\title{
Estimates of cancer burden in Italy
}

\author{
Silvia Rossi', Emanuele Crocetti2-3, Riccardo Capocaccia', Gemma Gatta4; \\ and the AIRTUM Working Group (see Appendix)
}

${ }^{1}$ National Center of Epidemiology, Italian National Institute of Health, Rome; ${ }^{2}$ Tuscany Cancer Registry, Cancer Prevention and Research Institute (ISPO), Florence; ${ }^{3}$ AIRTUM National Secretary;

${ }^{4}$ Fondazione IRCCS Istituto Nazionale dei Tumori, Milan, Italy

\section{ABSTRACT}

Aims and background. This paper presents updated estimates of the incidence, prevalence and mortality of stomach, colorectal, lung, breast, uterine cervix and prostate cancer and skin melanoma in the Italian population. In particular, point estimates for 2012 and time trends from 1970 to 2015 will be provided.

Methods. The presented figures were obtained by summing up the regional epidemiological indicator estimates presented in the other papers of this monographic issue, which were derived by applying the MIAMOD statistical back-calculation method to cancer registries survival data and official mortality rates.

Results. Our findings indicate that breast, colon-rectum and prostate will be the cancer sites with the highest incidence rates in the forthcoming years. The incidence rates still tend to increase for breast, male colorectal cancer and female lung cancer as well as for skin melanoma. By contrast, the incidence of stomach cancer, cervical cancer and male lung cancer, by far the most common tumor sites up to the early 1990s, will continue to decrease. The mortality estimates showed a decreasing trend for all considered cancers with the only exception of lung cancer in women.

Conclusion. These results point to the need to reinforce prevention activities by developing more effective preventive measures for population groups at risk. There is also a need to support timely and continuous cancer surveillance in the Italian population through cancer registries in order to monitor the spread of the cancer risk and to evaluate the impact of prevention policies and therapeutic advances.

\section{Introduction}

In Italy cancer is the second cause of death after cardiovascular disease, accounting for about one third of all deaths ${ }^{1}$. The National Health System, created in 1978 and mainly funded through taxation, is responsible for providing resident citizens with health care. The Italian National Health System is organized regionally and the Italian regions have to prepare periodic cancer control programs. However, the central government issues general policy indications for cancer control and makes available funds to the regions for their implementation. In order to build an appropriate knowledge-based strategy for cancer control, it is important to have available and continuously monitor quantitative measures of the disease burden, whose major indicators are incidence, mortality and prevalence.

Data on cancer mortality have been collected and made available in electronic format for the entire population by the Italian National Institute of Statistics (ISTAT) since 1970. Incidence data are collected by cancer registries and distributed by the Italian Network of Cancer Registries (AIRTUM) ${ }^{2}$. However, cancer registries data were available for one third of the national population in 2009, mostly from the north of the country. The coverage of the AIRTUM pool of cancer registries was $48 \%$ in the north of Italy, $26 \%$ in the center and $16 \%$ in the south ${ }^{3}$. Coverage increased from $3 \%$ in the early 1980 s to $15 \%$ in 1990 and $25 \%$ in 2000, up to the present proportion of $32 \%$. Cancer registries generally cover province-level areas and most have been active since the late $1980 \mathrm{~s}^{2}$.
Key words: cancer burden, incidence, Italy, prevalence, mortality.

Funding: The work presented in this paper has been partially funded by the "Programma Straordinario di Ricerca Oncologica 2006, Alleanza Contro il Cancro - Istituto Superiore di Sanità", project 2.4: "The Italian Cancer Registries Network", and by the project "Produzione e aggiornamento sistematico di stime a livello nazionale e regionale di alcuni tumori nella popolazione generale" funded by CCM, Italian Ministry of Health.

Conflict of interest statement: The authors declare no conflicts of interest. The funding sources had no role in study design, data collection, data analysis, data interpretation, writing this paper, or the decision to submit it for publication

Correspondence to: Silvia Rossi, National Center of Epidemiology, Istituto Superiore di Sanità, Viale Regina Elena 299, 00161 Rome, Italy. Tel +39-06-49904284; fax +39-06-49904285; email silvia.rossi@iss.it 
Statistical modeling is a useful complement to the field collection activity of cancer data. Models can be applied to empirical datasets with partial coverage deriving from cancer registry activity, to estimate epidemiological indicators with national or regional coverage and to project their estimated values up to the near future. In 2007, cancer incidence, mortality and prevalence data at the regional and national level were published in a monographic issue of Tumori ${ }^{4}$. The estimates were projected up to 2010 and were available for the 5 major cancer sites for people aged 0-84 years. The prevalence proportions have increased markedly, partly because of the improved survival of cancer patients and the aging of the population. In 2012, new and updated national epidemiological estimates and projections are clearly needed.

This paper will present updates of the basic epidemiological indicators (incidence, prevalence and mortality) for the 5 major cancers in Italy as described in the previous publication (lung, breast, prostate, colon-rectum and stomach) and will now include skin melanoma and cancer of the uterine cervix. In particular, point estimates for 2012 and time trends up to 2015 will be provided.

\section{Material and methods}

The incidence, mortality and prevalence estimates presented in this paper were obtained by summing up the absolute number of incident cases, deaths and prevalent cases, respectively, estimated for the 20 Italian regions and dividing them by the corresponding population data. Regional epidemiological indicator estimates, presented in the other papers of this monographic issue, were obtained by applying the MIAMOD statistical back-calculation method to survival and mortality data ${ }^{5-7}$. For further details we refer to the methods sections of these papers.

Briefly, the MIAMOD method was used for the estimation of incidence and prevalence. This statistical method is based on a back-calculation approach to estimate and project the morbidity of chronic irreversible diseases from mortality and patient survival. The method relies on the mathematical relationships between mortality, prevalence, incidence and survival. The model's estimation is based on mortality data from ISTAT for the period 1970-2002, with cause of death coded according to the ninth revision of the International Classification of Diseases (ICD-9) ${ }^{8}$. Relative survival data for the considered cancers for the period of diagnosis 1985-2002 were obtained from the EUROCARE-4 study'. The data refer to the populations covered by 21 cancer registries in Italy jointly covering about $25 \%$ of the national population.

Table 1 reports the size of the population observed by the Italian cancer registries in 2009 and the per- centage of coverage for each region and geographical area $^{3}$.

All incidence, mortality and prevalence estimates were carried out up to age 99 and for the period 1970-2015. For all regions, and therefore also in this paper, cervical cancer estimates were carried out only up to age 94 years because the distinction of uterus not otherwise specified (NOS) deaths into cervix and corpus uteri cancer deaths in women over 94 years old is not very reliable. Due to mortality data limitations in the past, only 15 -year prevalence is reported for cervical cancer. The age-standardized rates are based on the standard European population.

\section{Results}

The number of cases and both the crude and agestandardized incidence and mortality rates and prevalence proportions estimated in Italy for the year 2012 are presented in Tables $2 \mathrm{~A}$ and $2 \mathrm{~B}$, respectively, for men and women. In this year, colon and rectum was the most frequent cancer site, with over 54,000 new diagnoses in both sexes, followed by female breast, prostate and lung (50,396, 42,604 and 36,555 estimated incident cases, respectively). The incident cases for the other cancers were markedly lower, ranging between 13,560 (stomach in both sexes) and 1,515 (cervix) estimated new cases. The prevalence figures were highest (more than 10 times the incidence) for breast cancer. Prevalence was also high for colorectal and prostate cancer, but was substantially lower for the other considered cancer sites as a result of the poor prognosis of these cancers (lung, stomach) or the low incidence rates (melanoma, cervix). The highest crude mortality rates were for lung cancer in men (80 per 100,000 personyears) and breast cancer in women (35 per 100,000 person-years) and the lowest for melanoma in both sexes and cancer of the cervix uteri in women. For colorectal, lung, stomach cancer and melanoma, all the indicators were higher in men than women, with the exception of the melanoma prevalence. The highest male:female ratios for incidence and mortality were reported for lung cancer (3.6 for mortality and 3.2 for incidence), while the lowest male:female ratios were for melanoma (1.2 and 1.8 for incidence and mortality, respectively). The time trends of the 3 indicators over the period 19702015 are shown in Figures 1 to 6. They present the agestandardized incidence in men (Figure 1) and women (Figure 2), age-standardized mortality in men (Figure 3) and women (Figure 4), and crude prevalence in men (Figure 5) and women (Figure 6). The results will be described below, grouped by cancer site.

\section{Stomach}

The incidence and mortality trends of stomach cancer were estimated to be clearly decreasing during the 
whole period. Of those analyzed, stomach was the second most frequent cancer site in 1970, both in men (age-adjusted incidence: 51 per 100,000 person-years) and women (27 per 100,000 person-years). Projections to 2015 predict stomach cancer incidence as the least frequent cancer in men (17 per 100,000 person-years) and the second least frequent in women (9 per 100,000 person-years). The mortality trends were very similar to the incidence trends: the rates decreased from 44 to 11 per $100,000 /$ year in men and from 22 to 6 per $100,000 /$ year in women in the period 1970-2015. The prevalence proportions were slowly increasing in both sexes for the whole period, more in men than in women.

\section{Colon and rectum}

The age-standardized incidence rates of colorectal cancer were estimated to increase in males during the whole period up to a projected value of 71 per 100,000 personyears in 2015, even if the slope tended to flatten in the most recent years. In women the trend was similar but it preceded the male trend by several years. The rates in women were always lower than in men, and were first increasing and then slightly decreasing from 40 to 38 per $100,000 /$ year during the period 2004-2015. The mortality trends reflected the incidence trends with a more pronounced rate of decrease and with peak rates occurring about 20 years earlier. The estimated prevalence rose dramatically between 1970 and 2015: 10-fold in women and 15 -fold in men.

\section{Lung}

The lung cancer age-standardized incidence rates reached their peak in men during the late 1980s (94 per $100,000 /$ year) and decreased thereafter to an estimated value of 56 per 100,000 person-years in 2015. In women, the rates showed a linear increase from 8 per 100,000 person-years in 1970 to 20 per 100,000 person-years in 2015. Due to the very poor prognosis of this cancer, the mortality trends closely mimic the estimated incidence trends. In women, lung cancer was estimated to be the second most frequent cause of cancer death after breast cancer since 2010. If the present mortality trends will continue after 2015, lung cancer will become the most lethal cancer in females. The lung cancer prevalence is still low in women, due to its low incidence level in the past. By contrast, the epidemic peak observed in men during the second half of the 1980s is still reflected in increasing prevalence levels.

\section{Skin melanoma}

The melanoma incidence rates were estimated to increase for both genders. In men, we estimated a 13-fold increase from 1.6 to 21 per 100,000/year during the period 1970-2015, while in women the increase was lower (from 2 per 100,000 person-years in 1970 to 17 per
100,000 person-years in 2015). The trends were similar, with lower values in men until 2001, when the 2 genderspecific curves crossed over and the male rates increased more rapidly than the female rates. As a result, the agestandardized incidence is expected to be about $30 \%$ higher in men than women in 2015 . The mortality rates were low, due to the generally high survival of melanoma patients. They reached a peak in both genders during the 1990s and tended to decrease slightly thereafter. By contrast, the prevalence is substantial and is rapidly increasing, up to about 270 and 290 per 100,000 in 2015 in men and women, respectively.

\section{Breast}

The age-standardized incidence of female breast cancer in Italy was estimated to increase constantly from 43 per 100,000 person-years in 1970 to about 120 per 100,000 person-years in 2015. Trends and projections for breast cancer incidence from the 1990s onwards are to be taken with caution because this indicator reflects more the impact of screening activities than the natural evolution of risk factors. Our projections, based on observations up to 2002, foresee a continuing increase up to 2015, even though the rise seems to be less pronounced. The mortality rates reached a peak (29 per 100,000/year) by 1987 89 and then declined steadily to a foreseen level of 17 per 100,000 person-years in 2015 . The prevalence was estimated to rise markedly during the reporting period, and 2.5 out of 100 women are expected to live after a diagnosis of breast cancer in 2015.

\section{Cervix uteri}

The estimated age-standardized incidence and mortality rates for cervical cancer declined markedly during the period 1980-2015, reaching the lowest figures among the malignancies considered: 3.5 and 1.3 per 100,000 , respectively, in 2015 . The prevalence is also expected to decrease in the future down to an estimated 15-year prevalence of about 50 per 100,000 in 2015.

\section{Prostate}

The age-standardized incidence trend of prostate cancer was estimated to increase sharply, in particular during the period 1984-2005, from 31 per 100,000/year to 93 per 100,000/year. From 2005 onwards the rates seemed to stabilize or slightly decline to 89 per 100,000 person-years in 2015. The mortality for prostate cancer slightly increased from 16 per 100,000 person-years in 1970 to about 19 per 100,000 person-years in 1999 and then started to decrease, down to 14 per 100,000 person-years in 2015, with an estimated reduction of about $25 \%$ with respect to the 1999 peak level. Prevalence is estimated to rise consistently with increasing incidence up to reach a foreseen level of $1.2 \%$ of Italian men living in 2015 after a prostate cancer diagnosis. 


\section{Prevalence}

Prevalence increased for all considered cancers except cervix cancer. The rise was less pronounced in male and female stomach cancer due to the impressive reduction of its incidence, while it was striking for breast and prostate cancer. It is interesting to note the opposite behavior of mortality, which decreased for all considered cancers with the exception of lung cancer in women.

\section{Discussion}

We estimated the burden and trends for 7 major cancers in the Italian population, together with their time projections up to 2015. The work provides a 5-year update of similar data published in $2007^{4}$. Comparing the previously published estimates for the same age interval and including the common period 2000-2010 can provide useful insights into the stability of the results of this kind of estimation procedures and on possible uncertainties due to particular behaviors of specific cancers. Unfortunately, an exact direct comparison of the rates is not possible because the 2007 estimates are limited to the ages $0-84$ years. The effect of the different age range will be minimized by comparing age-standardized rather than crude rates. Furthermore, comparison of the general incidence and prevalence trends will by and large be indicative of possible inconsistencies between the 2 sets of estimates.

No relevant differences were found between the $2007^{4}$ and present estimates for stomach and colorectal cancer in both men and women. The estimates of the age-standardized incidence rates of stomach cancer were on average $5 \%$ lower for men and $15 \%$ lower for women than the present ones, as can be expected from the exclusion of ages $>85$ years, but the time trend was similarly decreasing. Also the age-standardized prevalence estimates were very similar both for men (old estimate 90 per 100,000 vs 89 per 100,000 according to the new estimate in 2010) and women (46 vs 48). For colorectal cancer we have very similar incidence figures, with an average annual percent change (APC) of $1 \%$ for men. For women, the new all-age incidence estimates including ages $>85$ years were about $4 \%$ higher than the old ones and the APC was close to zero in both series. The old and new estimated prevalence was again very similar (418 vs 408 per 100,000 for men and 270 vs 266 per 100,000 for women in 2010). The old male lung cancer incidence estimates were about $7 \%$ lower than the new ones, with a similarly decreasing trend $(-3 \%$ vs $-2.5 \%)$. For women, the present incidence estimates are higher than the old ones ( $14 \%$ more in 2000 and $28 \%$ more in 2010 ) and have a steeper rate of increase (APC $=0.6 v s 1.7)$. This indicates that the new data lead to a more pessimistic forecast of the future epidemic of lung cancer in Italian women. Accordingly, the new prevalence estimates were consistent with the old ones for men, but tended to be higher (by about one third in 2010) for women. The new estimates of the age-standardized breast cancer incidence rates are much higher than the old ones: from $+11 \%$ in 2000 to $+22 \%$ in 2010. This discrepancy cannot be due to missing ages $>85$ in the 2007 published data but is a real difference in the results, which also present an increasing incidence trend, with an APC of $1 \%$ per year instead of an almost flat trend in the old estimates. Even more different are the new estimates for prostate cancer, which can be compared only up to 2005, the last year provided in the old series. They are higher than the previous ones, as expected given the very high proportion of prostate cancers diagnosed at ages 85 or more, and have a much less steep rate of increase $(1.3 \% \mathrm{vs}$ $3 \%$ in the old estimate), mainly due to the effect of the very recent observations reported by cancer registries and the different modeling strategy adopted as a result.

Other meaningful comparisons can be made with the most recently published incidence trends based on the 1998-2005 observational data from the AIRTUM database $^{3}$. The southern regions are less well represented in the pool of Italian cancer registries than those of the north and center of the country. It can be expected that the lower representativeness is reflected in the differences between the AIRTUM data and this paper's estimates.

The stomach cancer incidence rates are relatively low in the southern regions, and this is consistent with our estimates being $10 \%$ lower in both sexes, with similar decreasing trends. For male colorectal cancer, the agestandardized incidence rates are close to each other and also the incidence trends are similar, increasing slightly during the 2000s. For females, our estimates are very similar to those of AIRTUM, with stable figures of around 40 per 100,000. The lung cancer trends are consistently decreasing in men and increasing in women, with $5 \%$ lower values for the Italian estimates with respect to those from the pool of registries. The national estimates behave similarly to the AIRTUM figures for melanoma, but are much lower for cervical cancer, particularly in the most recent years (5.3 vs 6.8 per 100,000 in 2005$)^{3}$. For prostate cancer the age-standardized incidence rates behave similarly up to the year 2000, after which our model estimates tend to slow while the AIRTUM figures continue to rise, even though at a lesser pace (92 vs 103 per 100,000 person-years, respectively, in 2005) ${ }^{3}$. For breast cancer the incidence trends are similar, still increasing in the same considered period (from 96 to 107 per 100,000/year in our estimates and from 105 to 115 per 100,000/year in the AIRTUM pool between 1998 and 2005), while an interesting difference was observed in the incidence rates: our national estimates are lower than the AIRTUM values. This can be partially due to the greater contribution of the southern population (with a lower breast cancer incidence) in the national estimates with respect to the AIRTUM pool of registries.

The differences between our national estimates and the AIRTUM estimates ${ }^{10,11}$ mainly depend on the fact that our Italian estimations were carried out considering data 
of all Italian regions, even those not covered by cancer registries, while the AIRTUM estimates were calculated considering only registry-covered areas. Therefore the lower concentration of registries in southern Italy reflects on the final outcome. According to the GLOBOCAN estimates, the Italian incidence rates for breast, prostate, colorectal and stomach cancer and skin melanoma are among the 20 highest in European countries ${ }^{12}$. In Italy, low incidence rates were reported for lung and cervix cancers. According to the CI5C incidence trends up to 2002, the tendency observed for Italy was similar to those reported for the other European countries ${ }^{13,14}$.

Projections of the time trends of the main epidemiological indicators are a key feature of the MIAMOD method. Knowledge of the cancer incidence and mortality trends is important for gaining a clear epidemiological picture of the changes that occur in our country in terms of diagnostic and therapeutic improvements, and therefore for defining priorities in health care planning and in resource allocation for cancer control programs. Furthermore, having at our disposal time trends for the future can be useful to suggest to policy makers geographical areas and types of interventions to contrast cancer.

The limitation of the MIAMOD method regards the projections for those tumors involved in screening programs, because the MIAMOD estimates are based on mortality data up to 2002 , which only partially reflect the effect of recent screening activities.
The estimates presented in this paper provide an indication of the cancer burden in Italy until 2015. Our findings indicate that breast, colon-rectum and prostate will be the cancer sites with the highest incidence rates in the forthcoming years. Conversely, the incidence of lung cancer, which was by far the most common cancer until the early 1990s, will continue to decrease, although only in men. The mortality estimates showed a decreasing trend for all studied cancers with the only exception of lung cancer in women.

These trends will produce a significant effect on the national hospitalization and care institutions. In a future perspective, because of the poor opportunities for the treatment of lung cancer, primary prevention through tobacco control is the priority, especially among women. For the reduction of colorectal cancer and breast cancer the main goal remains the dissemination of and compliance with organized screening programs.

These epidemiological findings support the need to intervene on the level of both prevention and health surveillance. On the one hand, it appears necessary to develop more effective preventive measures for population groups at risk of the damage of smoking (such as women and young people). On the other, it is necessary to maintain a timely and representative surveillance system of the Italian population through cancer registries to monitor the spread of the cancer burden and evaluate the impact of prevention policies and therapeutic advances.

Table 1 - Population observed by the Italian Network of Cancer Registries by region and geographical area and percentage of regional coverage in 2009

\begin{tabular}{lrr}
\hline Geographical area & Observed population in $2009(\mathrm{n})$ & Regional coverage in 2009 (\%) \\
\hline Piedmont & $1,088,710$ & 25.1 \\
Aosta Valley & 0 & 0.0 \\
Lombardy & $3,304,823$ & 35.2 \\
Trentino Alto Adige & 974,613 & 100.0 \\
Veneto & $2,294,685$ & 48.8 \\
Friuli Venezia Giulia & $1,204,718$ & 100.0 \\
Liguria & 875,732 & 55.0 \\
Emilia Romagna & $2,962,001$ & 71.3 \\
North of Italy & $12,705,282$ & 48.0 \\
Tuscany & $1,207,581$ & 33.6 \\
Umbria & 858,938 & 100.0 \\
Marche & 304,443 & 20.0 \\
Lazio & 519,850 & 9.9 \\
Center of Italy & $2,890,812$ & 25.7 \\
Abruzzo & 0 & 0.0 \\
Molise & 0 & 0.0 \\
Campania & $1,647,217$ & 28.5 \\
Puglia & 0 & 0.0 \\
Basilicata & 0 & 0.0 \\
Calabria & 0 & 0.0 \\
Sicily & $1,133,666$ & 22.6 \\
Sardinia & 469,870 & 28.5 \\
South of Italy & $3,250,753$ & 15.7 \\
Italy & $18,846,847$ & 32.2 \\
\hline
\end{tabular}


Table 2A - Estimated incidence, mortality and prevalence by cancer site for the year 2012 in Italy. Number of cases and deaths, crude and European age-standardized (age-std) rates per 100,000 person-years and crude prevalence proportion per 100,000 persons. Age 0-99 years, men.

\begin{tabular}{|c|c|c|c|c|c|c|c|c|}
\hline \multirow[b]{2}{*}{ Cancer site } & \multicolumn{3}{|c|}{ Incidence } & \multicolumn{3}{|c|}{ Mortality } & \multicolumn{2}{|c|}{ Prevalence } \\
\hline & $\begin{array}{c}\text { Number of } \\
\text { cases }\end{array}$ & $\begin{array}{l}\text { Crude } \\
\text { rate }\end{array}$ & $\begin{array}{l}\text { Age-std } \\
\text { rate }\end{array}$ & $\begin{array}{c}\text { Number of } \\
\text { deaths }\end{array}$ & $\begin{array}{l}\text { Crude } \\
\text { rate }\end{array}$ & $\begin{array}{l}\text { Age-std } \\
\text { rate }\end{array}$ & $\begin{array}{c}\text { Number of } \\
\text { cases }\end{array}$ & $\begin{array}{c}\text { Crude } \\
\text { proportion }\end{array}$ \\
\hline Prostate & 42,604 & 145.6 & 89.7 & 8,030 & 27.4 & 14.7 & 322,614 & $1,102.7$ \\
\hline Stomach & 7,879 & 28.8 & 18.2 & 5,272 & 19.3 & 11.8 & 37,981 & 138.9 \\
\hline Colon-rectum & 31,102 & 113.8 & 70.1 & 11,035 & 40.4 & 23.9 & 191,704 & 701.2 \\
\hline Lung & 26,334 & 96.2 & 60.7 & 21,984 & 80.3 & 49.5 & 63,499 & 232.0 \\
\hline Melanoma & 6,595 & 24.1 & 18.7 & 807 & 3.0 & 2.1 & 59,324 & 217.0 \\
\hline
\end{tabular}

Table 2B - Estimated incidence, mortality and prevalence by cancer site for the year 2012 in Italy. Number of cases and deaths, crude and European age-standardized (age-std) rates per 100,000 person-years and crude prevalence proportion per 100,000 persons. Age 0-99 years, women.

\begin{tabular}{|c|c|c|c|c|c|c|c|c|}
\hline \multirow[b]{2}{*}{ Cancer site } & \multicolumn{3}{|c|}{ Incidence } & \multicolumn{3}{|c|}{ Mortality } & \multicolumn{2}{|c|}{ Prevalence } \\
\hline & $\begin{array}{c}\text { Number of } \\
\text { cases }\end{array}$ & $\begin{array}{l}\text { Crude } \\
\text { rate }\end{array}$ & $\begin{array}{l}\text { Age-std } \\
\text { rate }\end{array}$ & $\begin{array}{c}\text { Number of } \\
\text { deaths }\end{array}$ & $\begin{array}{l}\text { Crude } \\
\text { rate }\end{array}$ & $\begin{array}{l}\text { Age-std } \\
\text { rate }\end{array}$ & $\begin{array}{c}\text { Number of } \\
\text { cases }\end{array}$ & $\begin{array}{c}\text { Crude } \\
\text { proportion }\end{array}$ \\
\hline Breast & 50,396 & 174.0 & 115.1 & 10,238 & 35.3 & 18.5 & 609,001 & $2,102.3$ \\
\hline Stomach & 5,681 & 19.6 & 9.3 & 3,884 & 13.4 & 6.1 & 28,158 & 97.2 \\
\hline Colon-rectum & 23,633 & 81.6 & 38.4 & 8,582 & 29.6 & 12.9 & 168,362 & 581.2 \\
\hline Lung & 10,221 & 35.3 & 19.0 & 7,780 & 26.9 & 13.8 & 25,504 & 88.1 \\
\hline Melanoma & 5,746 & 19.8 & 16.0 & 581 & 2.0 & 1.2 & 72,784 & 251.4 \\
\hline Cervix & 1,515 & 5.3 & 4.0 & 697 & 2.4 & 1.5 & $16,819 *$ & $58.9 *$ \\
\hline
\end{tabular}

*Limited-duration prevalence at 15 years.

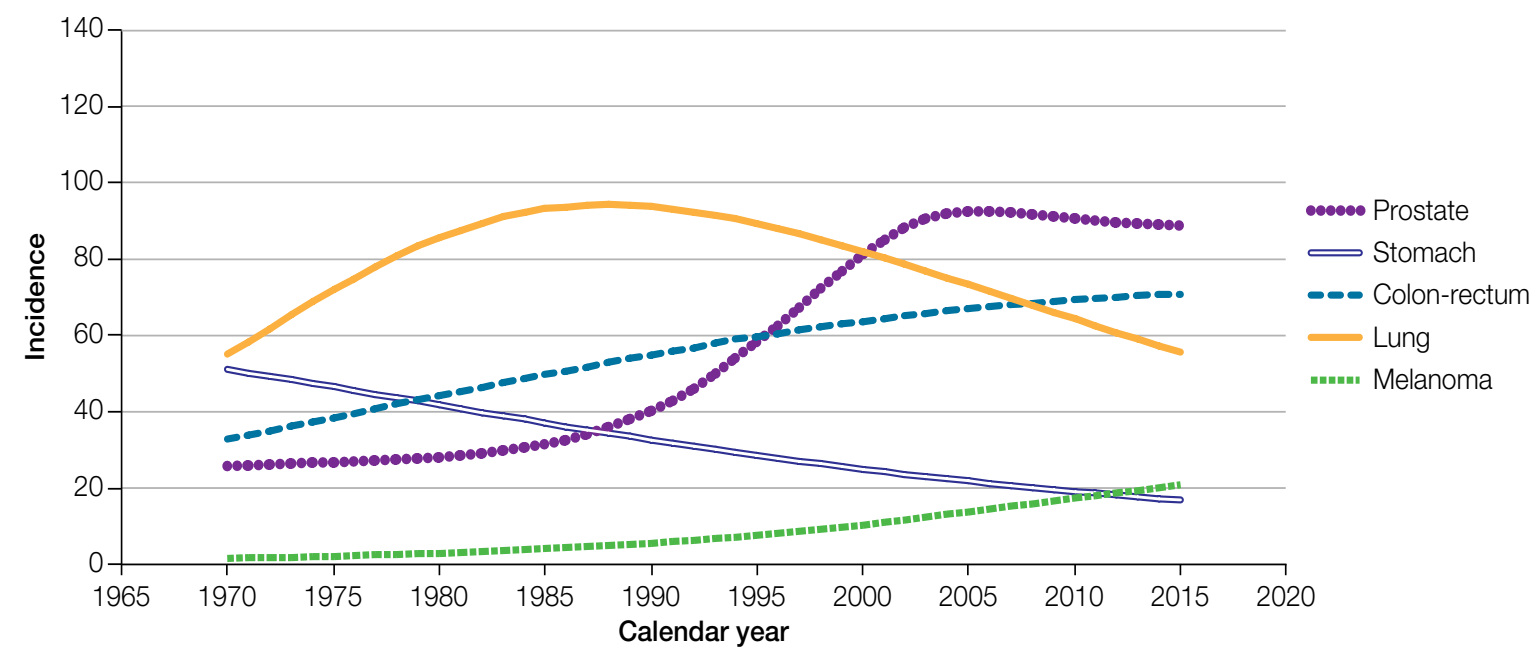

Figura 1 - Incidence estimates by cancer site in Italy in the period 1970-2015. Age-standardized rates (European population) per 100,000 personyears. Age $0-99$ years, men. 


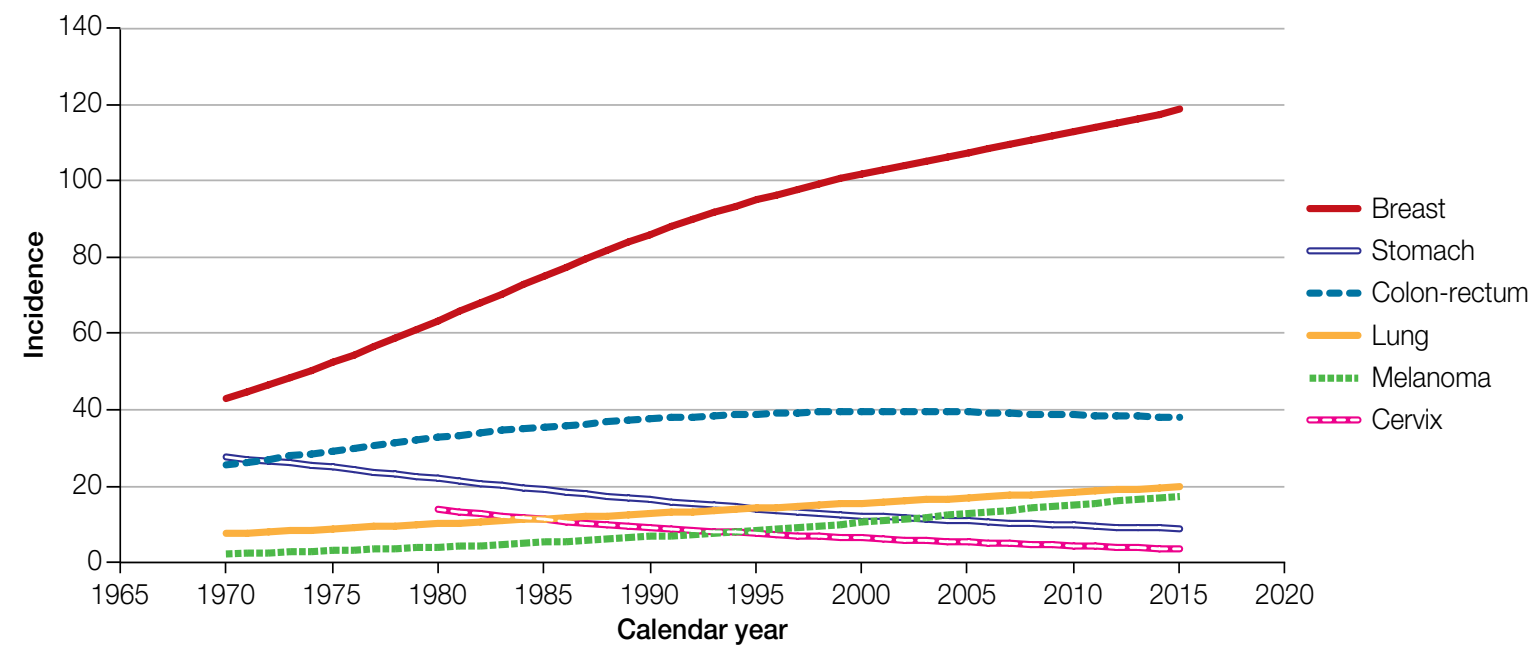

Figura 2 - Incidence estimates by cancer site in Italy in the period 1970-2015. Age-standardized rates (European population) per 100,000 personyears. Age 0-99 years, women.

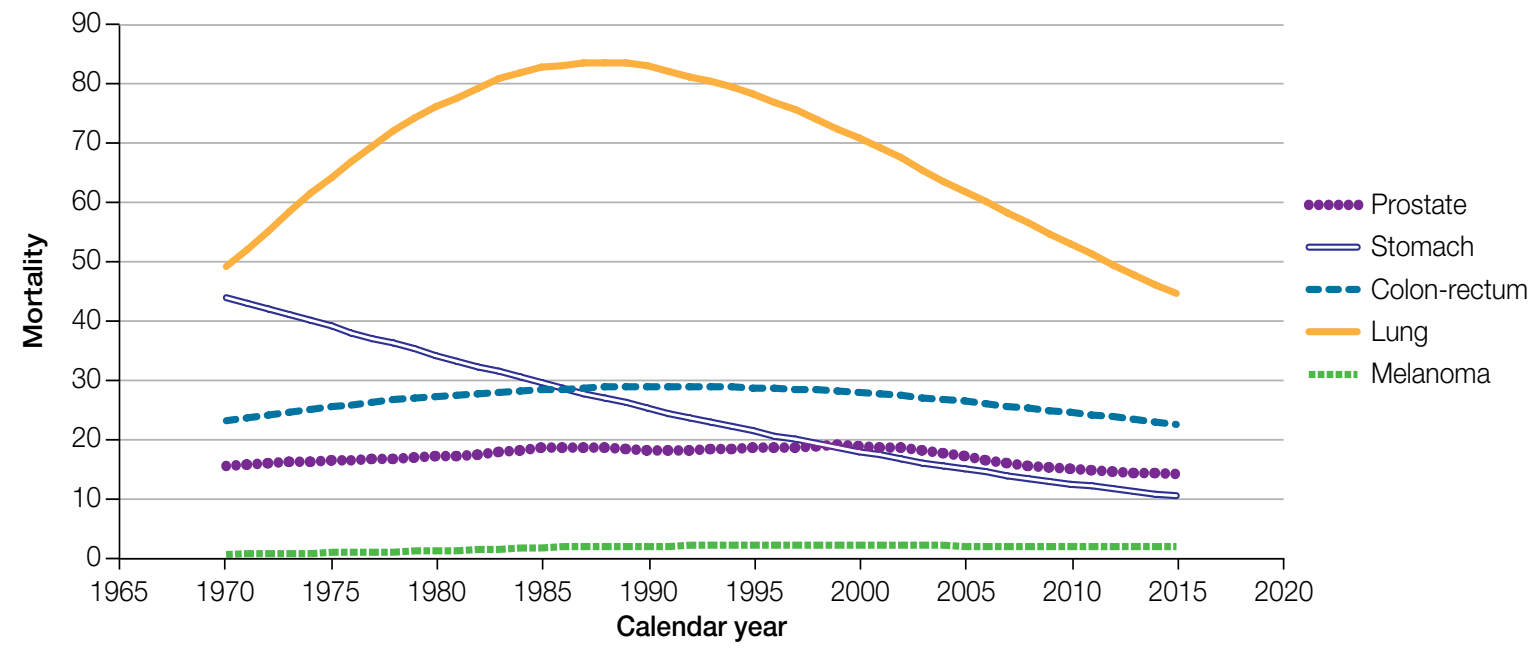

Figura 3 - Mortality estimates by cancer site in Italy in the period 1970-2015. Age-standardized rates (European population) per 100,000 personyears. Age $0-99$ years, men.

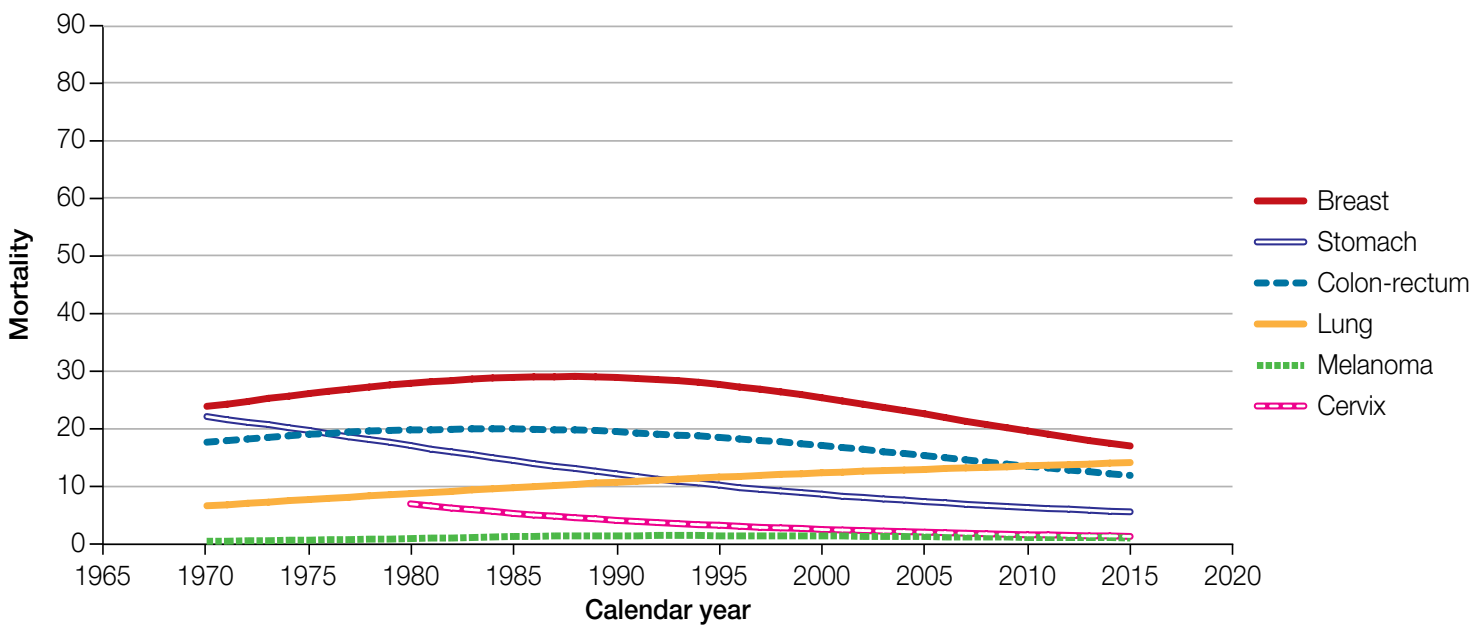

Figura 4 - Mortality estimates by cancer site in Italy in the period 1970-2015. Age-standardized rates (European population) per 100,000 personyears. Age 0-99 years, women. 


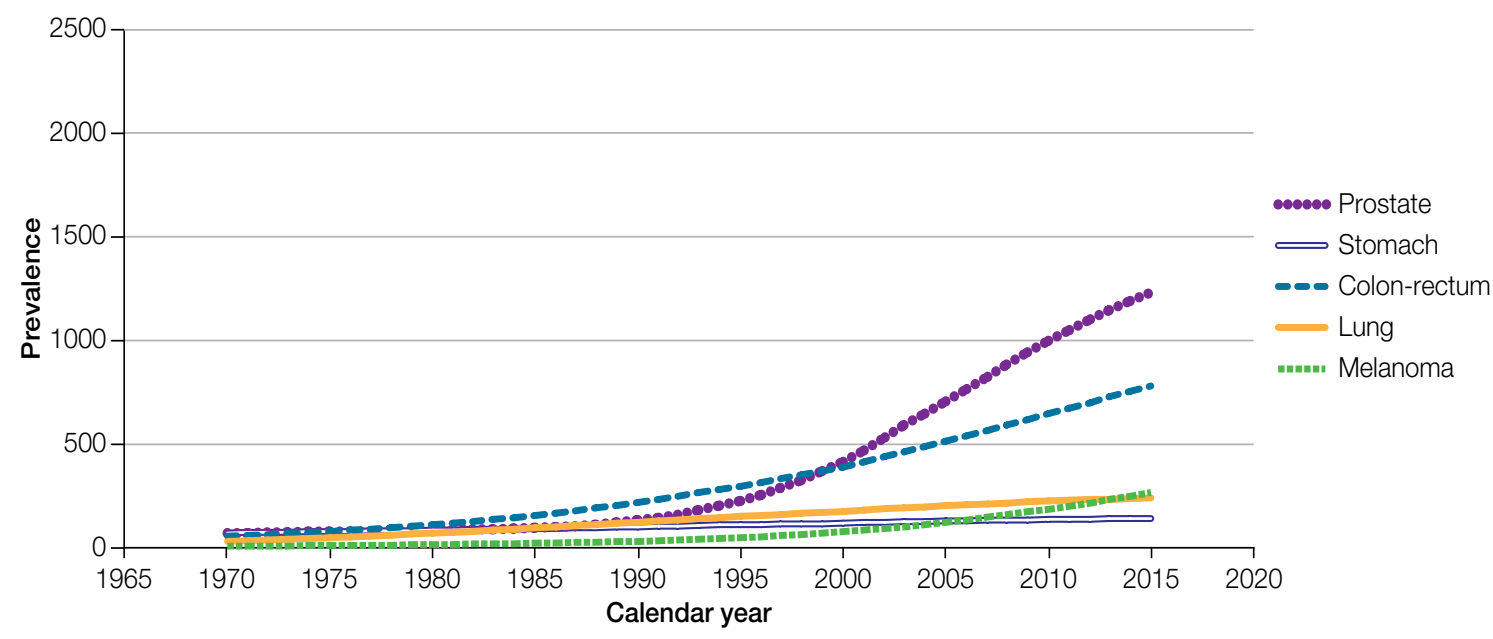

Figura 5 - Prevalence estimates by cancer site in Italy in the period 1970-2015. Crude proportion per 100,000 persons. Age 0-99 years, men.

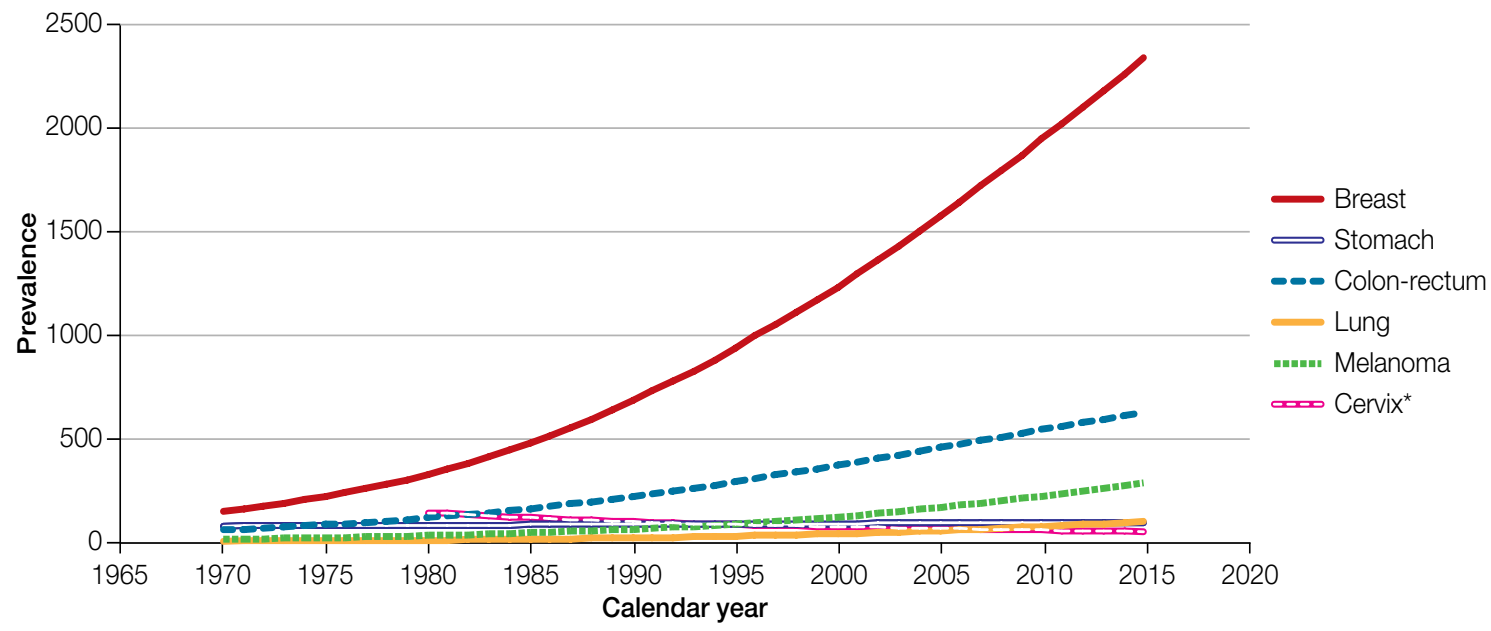

*limited-duration prevalence at 15 years

Figura 6 - Prevalence estimates by cancer site in Italy in the period 1970-2015. Crude proportion per 100,000 persons. Age 0-99 years, women.

\section{References}

1. ISTAT: Statistiche sulle cause di morte, anno 2008. http:// www.istat.it/dati/dataset/20110412_00/ (accessed 8 March 2013).

2. Italian Network of Cancer Registries (AIRTUM). http:// www.registri-tumori.it/cms/

3. AIRTUM Working Group: Italian cancer figures, report 2009: Cancer trend (1998-2005). Epidemiol Prev, 33 (4-5 Suppl 1): 1-168, 2009.

4. Micheli A, Francisci S, Baili P, De Angelis R: Current cancer profiles of the Italian regions. Tumori, 93: 329-407, 2007.

5. Verdecchia A, Capocaccia R, Egidi V, Golini A: A method for the estimation of chronic disease morbidity and trends from mortality data. Stat Med, 8: 201-206, 1989.

6. De Angelis G, De Angelis R, Frova L, Verdecchia A: MIAMOD: a computer package to estimate chronic disease morbidity using mortality and survival data. Comput Methods Programs Biomed, 44: 99-107, 1994.

7. Verdecchia A, De Angelis R, Francisci S, Grande E: Methodology estimation of cancer incidence, survival and prevalence in Italian regions. Tumori, 93: 337-344, 2007.

8. World Health Organization: International classification of diseases, 9th ed, WHO, Geneva, 1997.

9. Capocaccia R, Gavin A, Hakulinen T, Lutz JM, Sant M (Eds): Survival of cancer patients in Europe, 1995-2002: the EUROCARE-4 study. Eur J Cancer, 45: 901-1094, 2009.

10. AIRTUM: ITACAN. http://itacan.ispo.toscana.it (accessed 8 March 2013).

11. AIRTUM Working Group: Italian Cancer figures, report 2010 Cancer prevalence in Italy. Patients living with cancer, longterm survivors and cured patients. Epidemiol Prev, 34 (5-6) Suppl 2:1-187, 2010.

12. GLOBOCAN 2008: Cancer incidence, mortality and prevalence worldwide in 2008. http://globocan.iarc.fr/ (accessed 8 March 2013).

13. Curado MP, Edwards B, Shin HR, Storm H, Ferlay J, Heanue $\mathrm{M}$, Boyle P (Eds): Cancer incidence in five continents, Vol IX, IARC Scientific Publications No. 160, IARC, Lyon, 2007.

14. International Agency for Reaearch on Cancer: Cancer Incidence in Five Continents Annual Dataset. http://ci5. iarc.fr/CI5plus/ci5plus.htm (accessed 8 March 2013). 


\section{Appendix}

\section{AIRTUM Working Group}

C. Buzzoni (Banca Dati AIRTUM); A. Giacomin (Registro tumori della Provincia di Biella); R. Zanetti (Registro tumori Piemonte, città di Torino); L. Bisanti (Registro tumori Milano); R. Tessandori (Registro tumori di Sondrio); P. Crosignani (Registro tumori Lombardia, Provincia di Varese); M. Vercelli (Registro tumori Liguria, Provincia di Genova, UOS Epidemiologia descrittiva, IRCSS AOU SMIST/UNIGE); G. Mazzoleni (Registro tumori dell'Alto Adige-Tumorregister SüdTirol); S. Piffer (Registro tumori della Provincia di Trento); P. Zambon (Registro tumori del Veneto); D. Serraino (Registro tumori del Friuli-Venezia-Giulia); S. Ferretti (Registro tumori della Provincia di Ferrara); M. Michiara (Registro tumori della Provincia di Parma); M. Federico (Registro tumori della Provincia di Modena); M. Ponz de Leon (Registro dei tumori colorettali di Modena); L. Mangone (Registro tumori Reggiano); F. Falcini (Registro tumori della Romagna); E. Crocetti (AIRTUM National Secretary, Registro tumori Regione Toscana); F. La Rosa (Registro tumori umbro di popolazione); S. Vitarelli (Registro tumori di Macerata); F. Pannozzo (Registro tumori della Provincia di Latina); M. Fusco (Registro tumori di popolazione Regione Campania); A. Donato (Registro tumori della Provincia di Salerno); A. Traina (Registro tumori della Mammella di Palermo); R. Tumino (Registro tumori della Provincia di Ragusa); A. Madeddu and M.L. Contrino (Registro tumori di Siracusa); M. Budroni (Registro tumori della Provincia di Sassari). 